\title{
Going virtual: a report from the sixth Young Microbiologists Symposium on 'Microbe Signalling, Organisation and Pathogenesis'
}

\author{
Shi-qi An ${ }^{1, *}$, Bruno Silvester Lopes², James P. R. Connolly³ ${ }^{3}$ Connor Sharp ${ }^{4,5}$, Thi Kim Loan Nguyen ${ }^{6}$ and Clare \\ Louise Kirkpatrick ${ }^{7}$
}

\begin{abstract}
The sixth Young Microbiologists Symposium on 'Microbe Signalling, Organisation and Pathogenesis' was scheduled to be held at the University of Southampton, UK, in late August 2020. However, due to the health and safety guidelines and travel restrictions as a response to the COVID-19 pandemic, the symposium was transitioned to a virtual format, a change embraced enthusiastically as the meeting attracted over 200 microbiologists from 40 countries. The event allowed junior scientists to present their work to a broad audience and was supported by the European Molecular Biology Organization, the Federation of European Microbiological Societies, the Society of Applied Microbiology, the Biochemical Society, the Microbiology Society and the National Biofilms Innovation Centre. Sessions covered recent advances in all areas of microbiology including: Secretion and transport across membranes, Gene regulation and signalling, Host-microbe interactions, and Microbial communities and biofilm formation. This report focuses on several of the highlights and exciting developments communicated during the talks and poster presentations.
\end{abstract}

\section{INTRODUCTION}

The sixth Young Microbiologists Symposium (YMS) on 'Microbe Signalling, Organisation and Pathogenesis' was scheduled to be held at the University of Southampton, UK, on 26 and 27 August 2020.

A live meeting was cancelled due to the travel restrictions and strict health and safety guidelines put in place in the UK due to the COVID-19 pandemic. Rather than cancelling the planned meeting altogether, the organizers Shi-qi An (University of Southampton, UK), Clare Kirkpatrick (University of Southern Denmark, Denmark) and Bruno Silvester Lopes (University of Aberdeen, UK) worked to switch to a digital platform.

The transition to digital conferencing was enthusiastically embraced, with attendance of over 200 microbiologists from 40 countries in Asia, Australia, Europe, and North and South America. Most participants were junior principal investigators, postdoctoral researchers and students. The programme of 41 talks and 113 poster presentations provided junior scientists a platform to showcase their work, engage with their peers and foster collaborations.

As with the five previous meetings in this series, the virtual meeting had topical theme-based sessions discussing microbial intracellular signalling, antibiotic resistance, bacterial secretion and host-microbe interactions. Each session was topped-and-tailed by talks from renowned experts (a mix of invited junior and senior principal investigators), while the majority of the talks were given by young researchers from leading laboratories worldwide. Three keynote lectures featured eminent academics who have made substantial contributions to microbiology. There were also presentations from editors from various general science and microbiology journals in which the publication process was discussed.

The meeting highlighted the extraordinary range of functions associated with bacterial life, and emphasized recent

Received 10 November 2020; Accepted 06 January 2021; Published 02 February 2021

Author affiliations: ${ }^{1}$ School of Biological Sciences, National Biofilms Innovation Centre, University of Southampton, Southampton, UK; ${ }^{2}$ University of Aberdeen, Medical Microbiology, Aberdeen, UK; ${ }^{3}$ Newcastle University Biosciences Institute, Newcastle University, UK; ${ }^{4}$ Department of Biochemistry, University of Oxford, Oxford, UK; ${ }^{5}$ Department of Zoology, University of Oxford, Oxford, UK; ${ }^{6}$ Department of Microbiology, Humboldt University of Berlin, Berlin, Germany; 'Department of Biochemistry and Molecular Biology, University of Southern Denmark, Odense, Denmark.

*Correspondence: Shi-qi An, s-q.an@soton.ac.uk

Keywords: gene regulation; biofilm formation; antibiotic resistance; infection; host-pathogen interactions; signalling.

Abbreviations: AD, atopic dermatitis; CF, cystic fibrosis; eDNA, environmental DNA; MAC, membrane attack complex; RBD, receptor-binding domain;

scRNA-seq, single-cell RNA sequencing; T6SS, type VI secretion system; YMS, Young Microbiologists Symposium.

001024 (c) 2021 The Authors 


\section{Single-cell RNA-seq \& viral track}

COVID-19

Mild patient

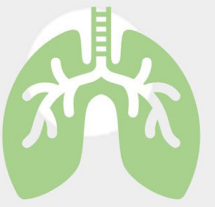

COVID-19

Severe patient

vS

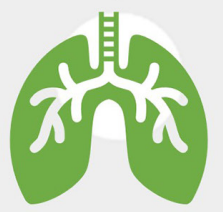

- Profile infected populations

- Detect co-infection

- Identify pathways specific to infected cells

\section{Antibody-based interventions against SARS- CoV-2 infection}

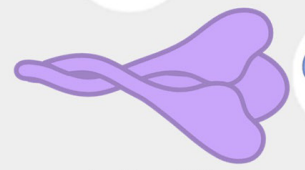

Spike (S) protein

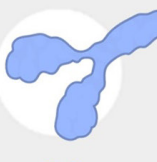

X

Binding

blocked

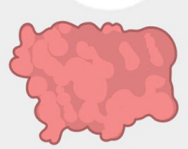

ACE2

receptor

Fig. 1. Schematic describing the structure-function experiments to examine SARS-CoV-2 infection.

discoveries regarding signalling and regulatory processes in bacterial development and virulence. In this report, we summarize the work presented in oral and poster presentations.

\section{SARS-COV-2 AND COVID-19: THE MOST IMPORTANT RESEARCH QUESTIONS}

Zheng Zhang (Southern University of Science and Technology, China) gave a timely special lecture on COVID-19, highlighting the recent discovery of immunopathogenesis and antibody responses elicited by SARS-CoV-2 infection and outlining some of the major questions currently being addressed (Fig. 1). In the first part of his talk, he described how single-cell RNA sequencing (scRNA-seq) is being used to characterize bronchoalveolar lavage fluid immune cells from patients with varying severity of COVID-19 and from healthy people [1]. He also demonstrated the application of Viral-Track, a computational method that globally scans un-mapped scRNAseq data for the presence of viral RNA. Analysis of sequencing data obtained from the bronchoalveolar lavage samples from COVID-19 patients revealed a dramatic impact of the virus on the immune system of severe patients compared to mild cases [2]. In the second part of his talk, he described isolation and characterization of receptor-binding domain (RBD)-specific monoclonal antibodies that potently neutralize SARS-CoV-2 [3]. Analysis of the crystal structure of RBD-bound antibody revealed that steric hindrance inhibits viral engagement with ACE2, thereby blocking viral entry. These findings suggest that anti-RBD antibodies are largely viral-species-specific inhibitors. The antibodies identified here may be candidates for development of clinical interventions against SARS-CoV-2 [3]. 


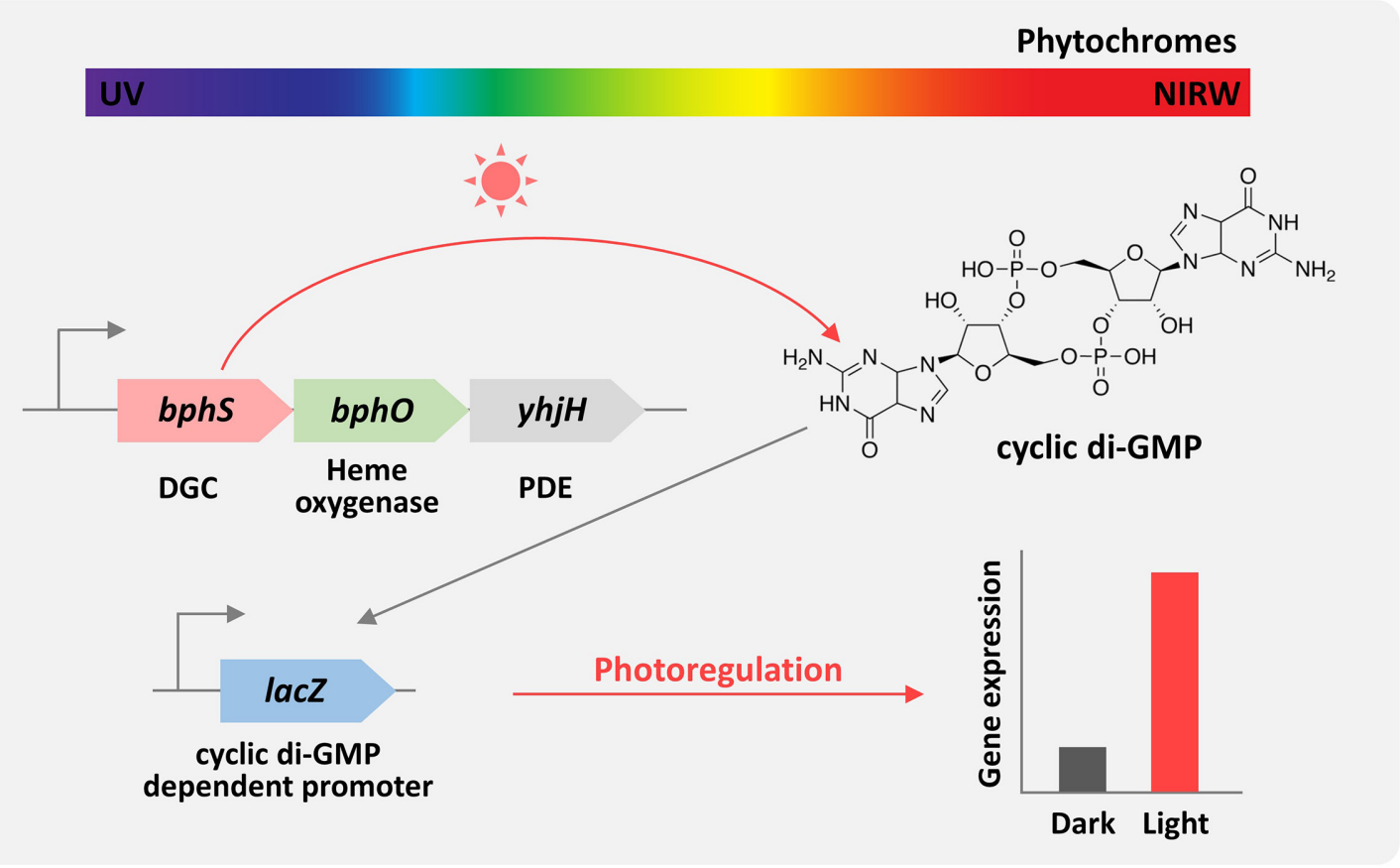

Fig. 2. Overview of the bacterial c-di-GMP-dependent photoregulatory module. Simplified schematic of the engineered NIRW-activated gene expression module, encoding the light responsive diguanylate cyclase (DGC) bphS, haem oxygenase bphO and phosphodiesterase (PDE) yhjH. Light activation generates c-di-GMP, which in turn activates expression of an engineered c-di-GMP-dependent reporter (in this case lacZ). Thus, light stimulation leads to 'photoregulation' of a target promoter via c-di-GMP production.

\section{SECRETION AND TRANSPORT ACROSS MEMBRANES}

The session on secretion and transport across membranes covered diverse aspects, including membrane remodelling which is forced upon the bacteria by the human innate immune system. Suzan Rooijakkers (University of Utrecht, the Netherlands) introduced the session with her group's work on how Gram-negative bacteria are killed by the complement system. The membrane attack complex (MAC) of the complement system has been well characterized in terms of its activity against eukaryotic cells or in cell-free model systems, but aspects of how it works against the double membrane of Gram-negative bacteria are still mysterious. Suzan's work has shed much light on this process by demonstrating that it is not sufficient for pre-assembled MAC complexes to attach to bacteria, but instead they must assemble directly on the outer membrane and be locally activated by convertase enzymes there [4]. Moreover, some pathogenic bacteria are able to resist the immune system by specifically interfering with this step [5].

In non-pathogenic Gram-negative bacteria, membrane and cell envelope remodelling is a critical step in cytokinesis. Chris Mahone from the Goley group (John Hopkins School of Medicine, USA) presented his work on the divisome of Caulobacter crescentus. Following up on the group's recent publication showing that the FtsZ-binding protein FzlA is needed for activation of the peptidoglycan synthase complex FtsWI [6], he could now show that the essential DNA translocase enzyme FtsK is also a part of this complex, thus linking chromosome segregation to completion of cell division.

Previous YMS meetings have featured the latest developments in our understanding of the type VI secretion system (T6SS), and this year was no exception. Patricia Bernal (University of Seville, Spain) presented her work on new structural components of the T6SS that were discovered through their interaction with the baseplate recruitment factor TssA [7]. TssA homologues can exist in short or long forms, but the reason for this diversity is not well understood. Patricia showed that the short TssA variants are consistently co-encoded with a short hypothetical protein, now named TagB, which is essential for T6SS sheath stabilization. In the absence of TagB, the tail and sheath structures of the T6SS were only half their normal length and the system could not 'fire' its effector proteins correctly. Moreover, the presence of short or long TssA isoforms was associated with different 'firing' behaviour of the T6SS (immediate or delayed, respectively), that was hypothesized to contribute to different behaviour of the bacteria depending on their environmental niche. The T6SS was also featured in two of the prize-winning hot-spot poster talks. Yoann Santin from the Cascales laboratory (CNRS Laboratoire d'Ingénierie des Systèmes Macromoléculaires, France) presented his group's work on the stabilizer of the 
Nutrition, immune system development, pathogen resistance

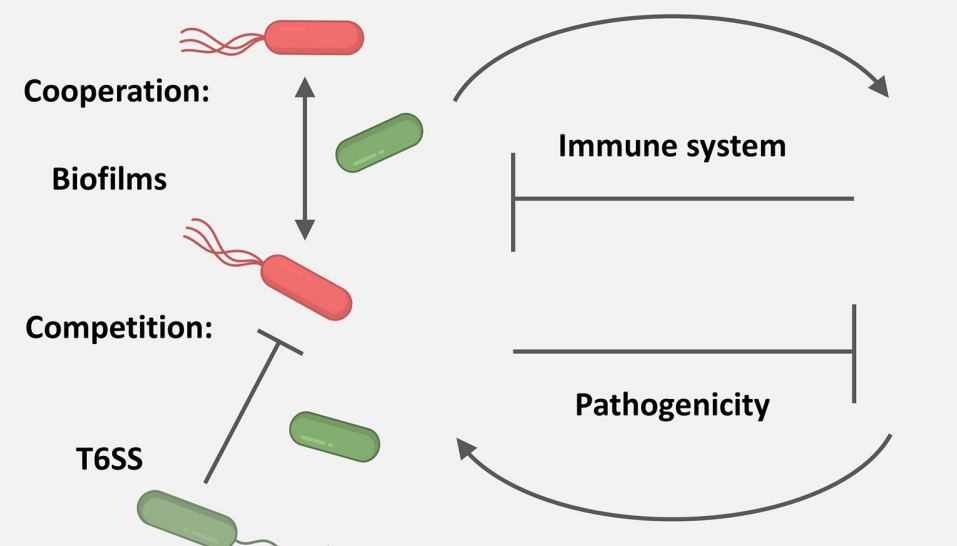

Stable conditions, nutrients

Fig. 3. The types of interactions between microbes and hosts

long TssA isoform, TagA [8], and how the tail length of the system is regulated by three-way contact between the end of the tail, TagA, and the membrane at the opposite side of the cell [9]. Meanwhile, Stephanie de Sousa (University of Sao Paulo, Brazil) presented a novel class of T6SS effector proteins with L,D-carboxypeptidase and L,D-transpeptidase activity, discovered in Salmonella. These effectors showed in vitro and in vivo activity against the peptidoglycan of target cells, suggesting that they may be employed against the native gut microbiota during Salmonella infection.

The theme was revisited during the keynote lecture of Lotte Søgaard-Andersen (Max Planck Institute, Marburg, Germany) on the second day, on the detailed characterization of the type IV pilus machine of Myxococcus xanthus. This complex structure spans the three layers of the Myxococcus xanthus cell envelope and enables the cells to carry out twitching motility by extending, adhering and retracting type IV pili through the machine in order to move along a surface. The core components of the machine were previously mapped by a highly innovative imaging approach combining cryo-electron tomography with systematic green fluorescent protein (GFP)-tagging of the machine components to precisely locate them in the complex and thereby infer how they contributed to the machine's overall function [10]. This lecture presented new structure-function data specifically on the hitherto unknown role of the minor pilins and the periplasmic protein PilY1 in the complex [11], combining biochemical and genetic analyses with similarly elegant imaging approaches to reveal their location and function at the pilus tip.

\section{GENE REGULATION AND SIGNALLING}

The second session featured diverse talks highlighting exciting novel mechanisms of cellular control and promising biomedical applications. The session was preceded by a special lecture from Mark Gomelsky (University of Wyoming, USA), a pioneer in the field of bacterial c-di-GMP-dependent signalling. He focused on work harnessing bacterial signalling modalities combined with the concept of photoreception for use in optogenetics. Bacteriophytochromes (Bph) allow bacteria to sense light in the near-infrared window (670$900 \mathrm{~nm}$ ). This is particularly attractive as this near-infrared light harmlessly penetrates mammalian tissue with minimal absorption and the Bph chromophore, biliverdin IXa, is naturally available in mammalian cells. The team developed a synthetic module for remote photocontrol of engineered cells, involving an engineered diguanylate cyclase that produces c-di-GMP in response to light and a phosphodiesterase that maintains background c-di-GMP noise in the absence of light (Fig. 2). Importantly, c-di-GMP is not produced by mammalian cells and so can be harnessed as an invisible 'orthogonal drug. Mark beautifully described how this system can be applied to model Escherichia coli cells and mammalian tissue of the mouse brain to mediate precise photocontrol of biological activity in vivo [12-14]

Continuing on the broad topic of c-di-GMP, Jacob Malone (John Innes Centre, Norwich, UK) presented an unusual mechanism of translational control by the soil bacterium Pseudomonas fluorescens. He gave a detailed overview of the rim $A B K$ operon, which encodes a c-di-GMP phosphodiesterase, poly-glutamate protease and an ATP-dependent 


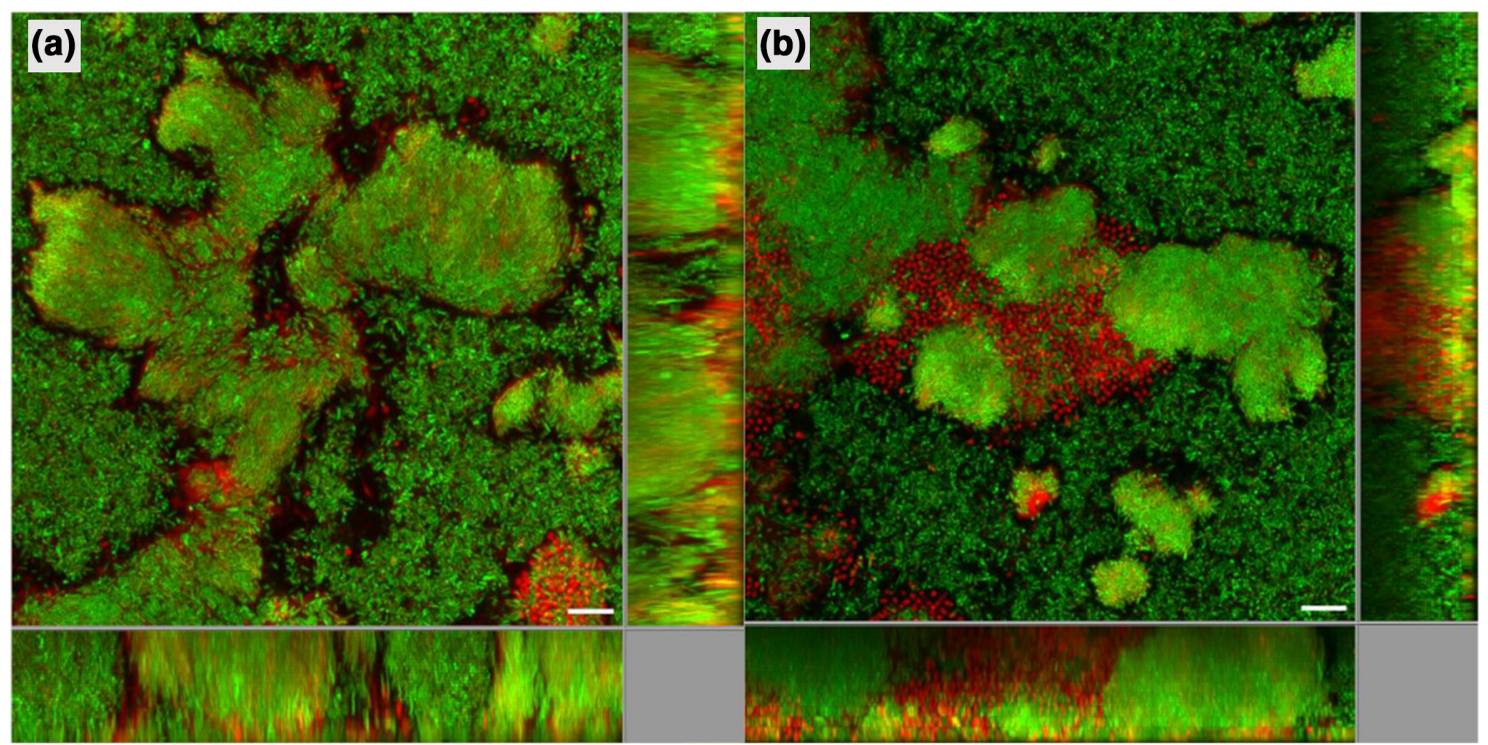

Fig. 4. Confocal images of biofilm cells. P. aeruginosa PAO1 (a) or $\triangle \mathrm{clpV1}$ (b) with Acinetobacter baumannii GFP-tagged P. aeruginosa live cells in green, and A. baumannii cells in red stained by Syto62. [Images reproduced with permission from Liang Yang (2019) npj Biofilms Microbiomes 5:1-11]

glutamyl ligase, respectively. RimK functions by adding glutamate residues to the ribosomal protein RpsF, which regulates the global proteome by influencing abundance of the RNA-chaperone Hfq and altering translation rates of target genes. RimA displays a dual functionality by directly interacting with RimK and degrading c-di-GMP. RimB ensures that glutamate chains on RpsF are formed in units of four. All three proteins interact, and their function maintains a fine balance of translational control in response to low temperature, nutrient deprivation and c-di-GMP concentration, which are known to regulate expression of the operon [15].

Elena Pajares-Martinez (University of Murcia, Spain) presented a fascinating story on the CarF desaturase of Myxococcus xanthus. CarF indirectly signals a light response enabling transcription of carotenoid biosynthesis genes and was found to produce plasmalogens, an abundant group of lipids important in animals but of unknown origin. Crucially, discovery of CarF function facilitated the identification of the human homologue TMEM189. This homologue could functionally replace CarF in bacterial cells and was essential for plasmalogen synthesis in human cells [16].

Veronica Urdaneta (Yale University, USA) gave an overview of a novel system developed to study the rules of chemical compound uptake and efflux in Pseudomonas aeruginosa, a common antibiotic-resistant human pathogen. The system is composed of a chromosomal lac $Z$ reporter fused to a specific riboswitch, which allows selective reporting of activity in response to a large compound library. By analysis of efflux-deficient reporter strains, the system can discriminate between compounds that are retained or removed from the cell. Proof of concept was demonstrated by analysis of folate-cycle-targeting drugs, which result in accumulation of ZMP/ZTP metabolites, thus activating the corresponding ZTP riboswitch specifically.

The final talk was given by Joan Geoghegan (University of Birmingham, UK) who described her work on understanding the molecular mechanisms associated with Staphylococcus aureus skin colonization of patients with inflammatory atopic dermatitis (AD). She focused on the characterization of key cell wall-anchored proteins, ClfB and FnbB, that promote specific adhesion of Staphylococcusureus to corneodesmosin ligands found on the surface of corneocytes that occur on the outermost surface of the epidermis of AD patients. Importantly, ClfB is highly expressed at the $\mathrm{pH}$ found on $\mathrm{AD}$ skin and binding is promoted by the low levels of natural moisturizing factor associated with $\mathrm{AD}$ skin [17-20].

\section{HOST-MICROBE INTERACTIONS}

The host-microbe interactions session covered a range of strategies by pathogens to avoid the immune system by manipulating host pathways that promote survival.

Pricille Brodin (Institut Pasteur, France) presented a study on the interactions of Mycobacterium ulcerans, the third most common Mycobacterium infection worldwide. Mycobacterium ulcerans is capable of producing severe lesions. However, these lesions are not accompanied by pain due to the secretions of a mycobacterial polyketide, mycolactone [21]. Rather than damaging neurons, the action of mycolactone is through a specific signalling pathway that causes 

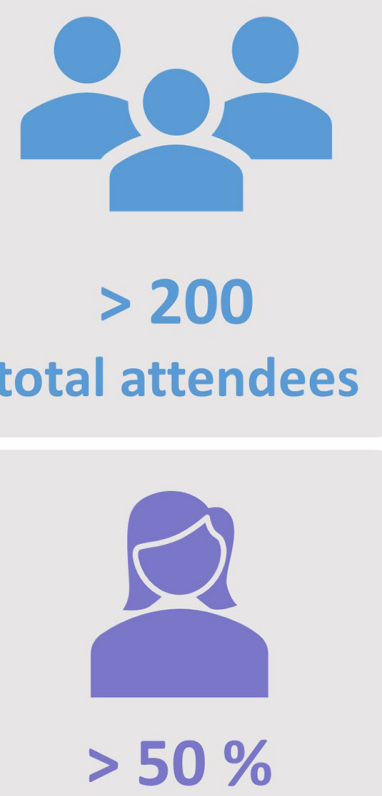

presenters are female

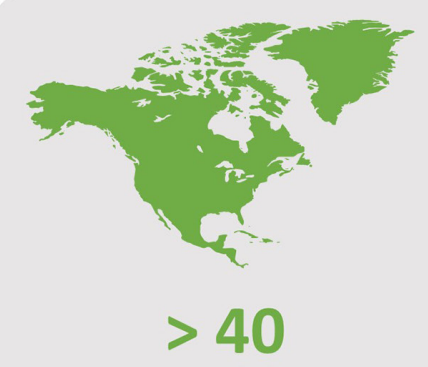

countries

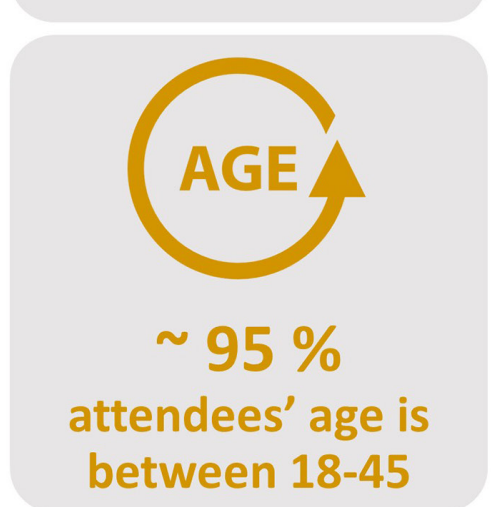

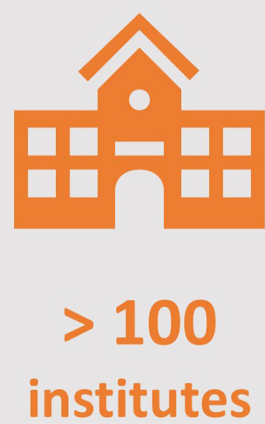

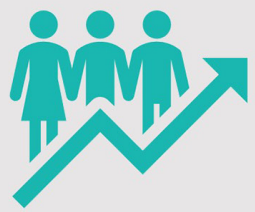

$>77 \%$

early career researchers

Fig. 5. Summary of meeting statistics associated with attendees and participants.

hyperpolarization. Using RNAi silencing, Pricille identified the receptor as $\mathrm{AT}_{2} \mathrm{R}$, which causes downstream activation of TRAAK potassium channels. Mycolactone and $\mathrm{AT}_{2} \mathrm{R}$ present a encouraging prospect for future analgesics.

Connor Sharp (University of Oxford, UK) focused on the evolution of the mutualistic relationship between hosts and the many bacteria of the microbiome. Theory has struggled to explain the evolution of a diverse microbiome where bacteria provide benefits to their host, as interbacterial competition should cause a collapse in mutualism (Fig. 3). Using evolutionary modelling he proposed that host control of the microbiome could explain the evolution of a diverse beneficial microbiome [22]. He then used a series of bioinformatic investigations to provide evidence for the key predictions of his model, focusing on the bacterial flagella as a marker for coevolution. Together these studies suggest a possible mechanism for the coevolution of mutualism in the microbiome.

Lisanne de Vor (Utrecht University, the Netherlands) described her work using monoclonal antibodies to target Staphylococcus aureus which can grow as a biofilm on surgical implants. In the first part of the talk, antibodies that had previously been shown to be effective at binding planktonic Staphylococcus aureus [23] were cloned into an IgG1 backbone, and tested for their ability to bind Staphylococcus aureus biofilms. An antibody which targets $\beta$-N-acetylglucosamine cell wall teichoic acid (anti-WTA) was found to have good binding to both polysaccharide-dependent and independent biofilms. Using indium 111 labelling, anti-WTA was demonstrated to localize with a biofilm-coated catheter in a mouse model. In the second part of the study, the ability of antiWTA antibodies to stimulate the complement system was assessed. Whilst the anti-WTA IgG1 antibody was capable of causing low levels of complement activation, engineering the same variable regions into a different IgG isotype was able to stimulate the complement system to a greater extent. Monoclonal antibodies could present a promising future treatment for Staphylococcus aureus biofilm infections.

To end the session, Liang Yang (Southern University of Science, China) gave a review of his extensive work in the field of biofilms in Pseudomonas aeruginosa healthcareacquired infections. Beginning with the physiological differences between planktonic and mono-species biofilms, Liang then examined multi-species biofilms where $P$. aeruginosa is able to dominate by upregulating its T6SS and polysaccharide production (Fig. 4) [24]. Chronic infection such as occurs in the cystic fibrosis (CF) lung drives selection in $P$. aeruginosa for biofilm formation, downregulation of quorum sensing, motility and a loss of pyoverdine production. A similar pattern is observed in shorter term infections during ventilator-associated pneumonia [25]. To finish, Liang explored $P$. aeruginosa co-infections with COVID-19 where biofilm formation may again be important for infections. 


\section{MICROBIAL COMMUNITIES AND BIOFILM FORMATION}

The fourth and final session of the meeting not only provided new insights into the development and control of biofilms in various bacterial species but also drew attention to the importance of the environmental microbiome on planetary health.

Brendan Gilmore (Queen's University Belfast, UK) kicked off the session with his talk on non-thermal 'cold' plasmas and their application for the control of biofilms. He showed that generating plasmas in air can give rise to a range of reactive oxygen and nitrogen species (RONS) and that $P$. aeruginosa biofilms initially exhibit a rapid decline in the number of surviving cells in the first few minutes upon exposure to plasma, followed by a significantly lower rate of cell number reduction [26]. Interestingly, biofilm architecture was not disrupted during this process, indicating that the presence of matrix increased tolerance against cold plasma in surviving cells. Additionally, he demonstrated that sub-lethal dosages of plasma can reduce the protective effect of matrix by enhancing the effect of antibiotics (plasma-antibiotic synergy), but it can also give rise to formation of persister cells.

Mary Turley (Trinity College Dublin, Ireland) presented her research on the effect of bile on biofilm formation of Staphylococcus aureus in a poster and talk. As Staphylococcus aureus is frequently isolated from CF patients suffering from aspiration of bile into the airways, she found that bile can induce biofilm formation in vitro. Further examination of multiple clinical isolates showed that these produced either protein- or polysaccharide-dependent biofilms or even both depending on the specific environmental conditions to which they were exposed.

Hannah Serrage (University of Bristol, UK), with a poster and talk, aimed to clarify the role of environmental DNA (eDNA) in oral biofilm formation. She first presented a novel image acquisition and analysis platform combined with widefield microscopy to reliably detect and quantify eDNA in biofilms. This allowed her to elucidate differences in structure and abundance of eDNA within the biofilm of not only Streptococcus gordonii, which she used as a model system, but also of several other single- and multi-species biofilms. She then beautifully illustrated how the contribution of different species to eDNA release can vary dependent on overall biofilm community structure.

Gabriele Berg (Graz University of Technology, Austria) highlighted the importance of the general environmental microbiome in the understanding of biofilms as well as its impact on planetary health. She used a range of plant model organisms to show how plant microbiomes can be shaped by several factors including breeding, domestication, storage and transportation. Finally, she emphasized how crucial the plant microbiome is for planetary health.

Marvin Whiteley (Georgia Institute of Technology, USA) closed the session with a special lecture on the quantification of the accuracy of various infection models. He questioned how well bacterial physiology within laboratory models would mimic chronic $P$. aeruginosa infections in human $\mathrm{CF}$ patients. By comparing corresponding transcriptome data and using a standard machine learning technique, his group successfully identified several genes whose expression reliably distinguished in vitro conditions from human infections [27] but also drew attention to potential drawbacks to this approach. He then continued to give insights into a novel and more robust, genome-wide framework that utilized so-called 'accuracy scores' that were calculated from mean expressions and standard deviations for each $P$. aeruginosa gene within target transcriptomes to aid experimentalists in selecting the appropriate infection model for their respective studies [28].

\section{SUMMARY}

The symposium brought much new insight in maintaining the track record of previous YMS meetings, and also provided an excellent forum for those with an interest in microbiology to interact and gain exposure to a wide range of topics. The lively and stimulating discussions between senior academics and early career scientists during oral and poster sessions clearly emphasized the quality of work on display. To get a flavour of the atmosphere of the meeting, see our short video overview here.

After the final keynote lecture, various awards were presented. Poster prizes were presented to Emma Banks (University of Nottingham, UK), Reuter Audrey (University Lyon, France), Susana Morcelle (National University of La Plata, Argentina) and Joanna Szczepaniak (University of Oxford, UK). In addition, oral presentation prizes were awarded to Stephanie de Sousa (University of Sao Paulo, Brazil), Katie Horton (University of Southampton, UK) and Yoann Santin (CNRS - Aix-Marseille Université, France).

There is no doubt that virtual conferences in the current time of COVID-19 raise challenges for organizers, attendees and speakers. The YMS was no exception but it highlighted a number of advantages. The relocation of the YMS to an online environment made it more accessible to a larger and more diverse audience, by-passing financial and visa restrictions that can often be barriers to attendance. Feedback from attendees was very positive, with participants appreciating the quality of the scientific programme and the atmosphere despite the constraints of a virtual environment. Our followup survey illustrated that $92.7 \%$ of the survey participants found the scientific programme 'good' or 'very good' and 96.4\% were interested in attending a future YMS conference. There were also some excellent suggestions for improvement and delivery of the next meeting (Fig. 5). This bodes well for another iteration of the meeting planned for 2022 and the possibility that it may be hybrid in nature.

\footnotetext{
Funding information

We are indebted to the European Molecular Biology Organization, Microbiology Society, Society for Applied Microbiology, Federation of European Microbiological Societies, National Biofilms Innovation Centre, Trends in Microbiology, Nature Reviews Microbiology, FEMS Microbes,
} 
Greiner Bio-One, New England Biolabs and all our other sponsors for their financial support.

\section{Acknowledgements}

The authors are grateful to the participants who agreed for their work to be described in this report and apologize to those whose work could not be mentioned due to space constraints. We also thank all the speakers, volunteers and participants for contributing to the success of this meeting. The authors are also indebted to Natasha Nater for her comments and input on this article, and support in organizing the meeting.

\section{Conflicts of interest}

The authors declare that there are no conflicts of interest.

\section{References}

1. Liao M, Liu Y, Yuan J, Wen Y, Xu G et al. Single-Cell landscape of bronchoalveolar immune cells in patients with COVID-19. Nat Med 2020;26:842-844

2. Bost P, Giladi A, Liu Y, Bendjelal Y, Xu G et al. Host-Viral infection maps reveal signatures of severe COVID-19 patients. Cell 2020;181:e12:1475-1488.

3. Ju B, Zhang Q, Ge J, Wang R, Sun J et al. Human neutralizing antibodies elicited by SARS-CoV-2 infection. Nature 2020;584:115-119.

4. Heesterbeek DA, Bardoel BW, Parsons ES, Bennett I, Ruyken M et al. Bacterial killing by complement requires membrane attack complex formation via surface-bound C5 convertases. Embo J 2019:38:e99852.

5. Doorduijn DJ, Bardoel BW, Heesterbeek DAC, Ruyken M, Benn G et al. Bacterial killing by complement requires direct anchoring of membrane attack complex precursor C5b-7. PLoS Pathog 2020;16:e1008606.

6. Lariviere PJ, Mahone CR, Santiago-Collazo G, Howell M, Daitch AK et al. An essential regulator of bacterial division links FtsZ to cell wall synthase activation. Curr Biol 2019:29:1460-1470.

7. Planamente S, Salih O, Manoli E, Albesa-Jové D, Freemont PS et al. TssA forms a gp6-like ring attached to the type VI secretion sheath. Embo J 2016:35:1613-1627.

8. Santin $Y G$, Doan $T$, Lebrun $R$, Espinosa $L$, Journet $L$ et al. In vivo TssA proximity labelling during type $\mathrm{VI}$ secretion biogenesis reveals TagA as a protein that stops and holds the sheath. Nat Microbiol 2018;3:1304-1313.

9. Santin YG, Doan T, Journet L, Cascales E. Cell width dictates type VI secretion tail length. Curr Biol 2019;29:3707-3713.

10. Chang Y-W, Rettberg LA, Treuner-Lange A, Iwasa J, SøgaardAndersen $L$ et al. Architecture of the type IVA pilus machine. Science 2016;351:aad2001.

11. Treuner-Lange A, Chang Y-W, Glatter T, Herfurth M, Lindow S et al. PilY1 and minor pilins form a complex priming the type IVA pilus in Myxococcus xanthus. Nat Commun 2020:11:1-14.

12. Ryu M-H, Gomelsky M. Near-Infrared light responsive synthetic c-di-GMP module for optogenetic applications. ACS Synth Biol 2014:3:802-810.

13. Ryu M-H, Kang I-H, Nelson MD, Jensen TM, Lyuksyutova Al et al. Engineering adenylate cyclases regulated by near-infrared window light. Proc Natl Acad Sci U S A 2014;111:10167-10172.
14. Fomicheva A, Zhou C, Sun Q-Q, Gomelsky M. Engineering adenylate cyclase activated by near-infrared window light for mammalian optogenetic applications. ACS Synth Biol 2019:8:1314-1324.

15. Grenga L, Little RH, Chandra G, Woodcock SD, Saalbach G et al. Control of mRNA translation by dynamic ribosome modification. PLoS Genet 2020;16:e1008837.

16. Gallego-García A, Monera-Girona AJ, Pajares-Martínez E, BastidaMartínez E, Pérez-Castaño $\mathrm{R}$ et al. A bacterial light response reveals an orphan desaturase for human plasmalogen synthesis. Science 2019;366:128-132.

17. Fleury OM, McAleer MA, Feuillie C, Formosa-Dague C, Sansevere $\mathrm{E}$ et al. Clumping factor B promotes adherence of Staphylococcus aureus to corneocytes in atopic dermatitis. Infect Immun 2017;85:e00994-16.

18. Feuillie C, Vitry P, McAleer MA, Kezic S, Irvine AD et al. Adhesion of Staphylococcus aureus to corneocytes from atopic dermatitis patients is controlled by natural moisturizing factor levels. mBio 2018:9:e01184-18.

19. Vitry P, Valotteau C, Feuillie C, Bernard S, Alsteens D et al. Forceinduced strengthening of the interaction between Staphylococcus aureus clumping factor $B$ and loricrin. mBio 2017:8:e01748-17.

20. Lacey KA, Mulcahy ME, Towell AM, Geoghegan JA, McLoughlin RM. Clumping factor $B$ is an important virulence factor during Staphylococcus aureus skin infection and a promising vaccine target. PLoS Pathog 2019;15:e1007713.

21. Marion E, Song O-R, Christophe T, Babonneau J, Fenistein D et al. Mycobacterial toxin induces analgesia in Buruli ulcer by targeting the angiotensin pathways. Cell 2014;157:1565-1576.

22. Foster KR, Schluter J, Coyte KZ, Rakoff-Nahoum S. The evolution of the host microbiome as an ecosystem on a leash. Nature 2017:548:43-51.

23. Lehar SM, Pillow T, Xu M, Staben L, Kajihara KK et al. Novel antibody-antibiotic conjugate eliminates intracellular S. aureus. Nature 2015:527:323-328.

24. Cheng Y, Yam JKH, Cai Z, Ding Y, Zhang L-H et al. Population dynamics and transcriptomic responses of Pseudomonas aeruginosa in a complex laboratory microbial community. NPJ Biofilms Microbiomes 2019:5:1-11.

25. Wang T, Qi Y, Wang Z, Zhao J, Ji L et al. Coordinated regulation of anthranilate metabolism and bacterial virulence by the GntR family regulator MpaR in Pseudomonas aeruginosa. Mol Microbiol 2020;114:857-869.

26. Alkawareek MY, Algwari QT, Laverty G, Gorman SP, Graham WG et al. Eradication of Pseudomonas aeruginosa biofilms by atmospheric pressure non-thermal plasma. PLoS One 2012;7:e44289.

27. Cornforth DM, Dees JL, Ibberson CB, Huse HK, Mathiesen IH et al. Pseudomonas aeruginosa transcriptome during human infection. Proc Natl Acad Sci U S A 2018;115:E5125-E5134.

28. Cornforth DM, Diggle FL, Melvin JA, Bomberger JM, Whiteley M. Quantitative framework for model evaluation in microbiology research using Pseudomonas aeruginosa and cystic fibrosis infection as a test case. mBio 2020;11:e03042-19.

\section{Five reasons to publish your next article with a Microbiology Society journal}

1. The Microbiology Society is a not-for-profit organization.

2. We offer fast and rigorous peer review - average time to first decision is 4-6 weeks.

3. Our journals have a global readership with subscriptions held in research institutions around the world.

4. $80 \%$ of our authors rate our submission process as 'excellent' or 'very good'

5. Your article will be published on an interactive journal platform with advanced metrics.

Find out more and submit your article at microbiologyresearch.org. 